\title{
Shedding New Lights with the Breakthrough Ideas to Understand Current Trends in Modern Toxicology
}

\author{
Ok-Nam Bae ${ }^{1}$ and Joo Young Lee ${ }^{2}$ \\ 'College of Pharmacy, Institute of Pharmaceutical Sciences, Hanyang University, Ansan, Korea \\ ${ }^{2}$ Integrated Research Institute of Pharmaceutical Sciences, College of Pharmacy, \\ The Catholic University of Korea, Bucheon, Korea
}

(Received January 13, 2016; Accepted January 14, 2016)

Along with the development of cutting-edge technologies in biology and chemistry, the scope and the criteria in toxicological research have been rapidly expanding (1). Under the main theme of 'New Frontier in Modern Toxicology', the $7^{\text {th }}$ International Congress of Asian Society of Toxicology (ASIATOX 2015) at Jeju Island in Korea on June 2015 provided a platform to talk about the current topics in toxicology. Recent progresses in translational, mechanistic and predictive toxicological research have been extensively discussed in various aspects including methodological, conceptual, or practical approaches.

To share the scientific progresses and to increase the toxicological impact of the contents discussed in ASIATOX 2015, the editorial board of the Toxicological Research, the official journal of the Korean Society of Toxicology who co-hosted the congress with the Asian Society of Toxicology, decided to publish an ASIATOX2015 special issue, 'Understanding of current trends in modern toxicology'. We believe that this special issue will help the readers keep up with the recent advances in breakthrough ideas in modern toxicology.

In this special issue, ten outstanding speakers in ASIATOX2015 presented their opinion in forms of either reviews or original articles, from various points of view in different toxicological research field. Among ten papers in this special issue, first three review papers are addressing the important paradigm shift in toxicological studies. In 'Advancing Risk Assessment through the Application of Systems Toxicology' by Sauer et al., authors discussed the current state of systems toxicology and the potential for its

\footnotetext{
Correspondence to: Joo Young Lee, College of Pharmacy, The Catholic University of Korea, Bucheon 420-743, Korea E-mail: joolee@catholic.ac.kr
}

This is an Open-Access article distributed under the terms of the Creative Commons Attribution Non-Commercial License (http:// creativecommons.org/licenses/by-nc/3.0) which permits unrestricted non-commercial use, distribution, and reproduction in any medium, provided the original work is properly cited. future application in risk assessment (2). To accomplish more predictive and accurate approaches to risk assessment, systems toxicology enables the integration of quantitative systems-wide molecular changes linking chemical exposures with adverse outcomes. These quantitative translational safety testing strategies and the related recent advances are outlined in the review. In the $2^{\text {nd }}$ paper titles as 'Advances in the Development and Validation of Test Methods in the United States', Dr. Casey from the US National Toxicological Program provided an overview of recent activities of interagency collaboration in development of alternative toxicological methods (3). The needs for alternative tests are now increasing for better understanding of toxicity pathways in predictive testing strategies with a focus on replacing, reducing, or refining animal use (4). The examples of alternative testing methods are included in the review to enhance the understanding of the application of alternative tests in the regulatory safety testing. In the $3^{\text {rd }}$ paper of 'Addressing Early Life Sensitivity using Physiologically Based Pharmacokinetic Modeling and In Vitro to In Vivo Extrapolation' by Yoon and Clewell, the authors described the process of in vitro and in vivo extrapolation (IVIVE) to develop physiologically based pharmacokinetic (PBPK) models, especially focused on a susceptible population such as children (5). A link between the concept of adverse outcome pathway (AOP) and the translational PBPK models has been provided, emphasizing the importance of PBPK models in predicting the age-specific target tissue exposure using in vitro/in silico approaches. PBPK modeling approaches using IVIVE and a case study for early life PBPK modeling of pyrethroids are included. These three reviews by opinion leaders in each field provide important insights into the goal of toxicity testing in modern toxicology, and into the way how we can efficiently and accurately understand the mechanism-based and quantitative relationship between chemical exposure and adverse outcome in human health.

Following the overview on current trends in the toxicological testing and risk assessment, three papers on target- 
organ toxicity are listed in this issue. In $4^{\text {th }}$ paper titled as ' $A$ Review of Toxicity and Carcinogenicity of Dichlorodiphenyltrichloroethane (DDT) with Special Reference to Hepatocarcinogenesis', Harada et al. extensively discussed hepatotoxicity and hepatic tumor development in liver induced by DDT exposure (6). Although DTT has been well-known to induce neurotoxicity and endocrine disruption, the review brought up a new aspect to look into other target organ toxicity of DTT by delineating the mechanistic basis of hepatic toxicity such as hepatic microsomal enzyme expression, oxidative stress, cell proliferation, and gap junction intercellular communication. Along with this paper, the $5^{\text {th }}$ paper in this issue is also dealing with hepatotoxicity. Chun et al. provided a comprehensive review on ischemic liver damage and the underlying mechanisms in 'Autophagy in Ischemic Livers: A Critical Role of Sirtuin 1/Mitofusin 2 Axis in Autophagy Induction' (7). Basic concepts for hepatotoxicity and ischemic/reperfusion (I/R) injury in liver have been described in detail, giving an introductive information to the readers. More importantly, recent progresses in mechanistic explanations for liver I/R injury have been included focusing on the role of autophagy. The pathological importance, signaling pathways, and the related terms in autophagy are outlined. The authors provided new insights into the significance of mitophagy and its central regulator sirtuins in hepatic I/R injury. In the $6^{\text {th }}$ article of 'Pyruvate Kinase M2: a Novel Biomarker for the Early Detection of Acute Kidney Injury' by Cheon et al. authors overviewed the predictive biomarkers for nephrotoxicity (8). While the urgent need for accurate and sensitive prediction of chemical-associated acute kidney injury (AKI) exists, the clinically acceptable biomarkers for early detection of AKI are limited. The authors provided a full review on currently proposed biomarkers for early detection of AKI, along with the advantages and the limitation of these biomarkers. Interestingly, the authors included their recent findings on pyruvate kinase M2 (PKM2), suggesting PKM2 as a potential candidate for early biomarker to detect AKI based on the results from metabolomic analysis. The innovative ideas in traditional toxicological topics of hepato/nephrotoxicity, such as integrative evaluation of hepatocarcinogenicity of DDT, new mechanistic pathway of mitophagy in hepatic I/R injury, and the use of omics strategy to explore mechanism-based nephrotoxic biomarkers, are giving important lessons to the future direction for mechanistic and translational toxicological studies.

In the last part of the special issue, four papers on the toppriority toxic chemicals are included. The exposure to heavy metals has long been considered to exert a greatly detrimental impact on human health. Three articles in this issue delineated the link between the exposure to heavy metals and the potential human risks. In the $7^{\text {th }}$ paper of 'Association between Multiple Metal Exposure and Renal Impairment in Korean Population', Lim et al. analyzed the relationship between the environmental exposure levels of heavy metals $(\mathrm{Pb}$ and $\mathrm{Cd})$ and toxicity markers in 1,953 adults and found that there existed the synergism of the renal tubular impairment outcome by exposure to multiple metals (9). These provide critical clues to be considered for risk assessment of multiple heavy metal exposure. In $8^{\text {th }}$ paper titled as ' $\mathrm{Cad}$ mium Exposure and Potential Health Risk from Foods in Contaminated Area, Thailand', Chunhabundit assessed the current contamination status of $\mathrm{Cd}$ in foodstuffs derived from the polluted area of Thailand and suggested the potential health risks of high $\mathrm{Cd}$ exposure from food in the region (10). These articles provide important information on the degree of heavy metal exposure in Asian countries and the association of environmental and daily exposure with the impact on the development of human diseases. The $9^{\text {th }}$ paper titled as 'Different Regulation of p53 Expression by Cadmium Exposure in Kidney, Liver, Intestine, Vasculature, and Brain Astrocytes' by Lee et al. delineated the mechanism as to how chronic exposure of heavy metal led to target organ toxicity using 12-month exposure study in mice together with cell line system (11). The accumulation of p53 in kidney and the induction of apoptosis were evident in kidney and well correlated with impairment of renal function. The article suggested that there may be cellular diversity in response to $\mathrm{Cd}$ depending on tissue type. In the last $10^{\text {th }}$ paper of ' $A$ Rapid and Sensitive Detection of Aflatoxin-Producing Fungus using an Optimized Polymerase Chain Reaction (PCR)', Bintvihok et al. developed a new method to detect contamination of aflatoxin-producing fungus in foods (12). Contaminated toxic compounds in food are still a severe threat against public health and rapid and sensitive detection of toxic chemicals or responsible bioorganisms are essential part for regulatory toxicity. In this research paper, authors newly developed a simple and sensitive method using real-time PCR to detect fungus producing aflatoxin that is a potent hepatocarcinogen. Metals and food-associated carcinogens are attracting continuous attention in public health, and these papers are providing recent progresses on toxic chemicals of regulatory concern.

In summary, this special issue of the journal provides valuable reviews and research articles, in an attempt to enhance scientific insights in the outstanding ideas discussed in ASIATOX 2015. We expect that this special issue covering the various fields of toxicological research helps us better understand the current status of modern toxicology.

\section{REFERENCES}

1. Rouquié, D., Heneweer, M., Botham, J., Ketelslegers, H., Markell, L., Pfister, T., Steiling, W., Strauss, V. and Hennes, C. (2015) Contribution of new technologies to characterization and prediction of adverse effects. Crit. Rev. Toxicol., 45, 172-183.

2. Sauer, J.M., Kleensang, A., Peitsch, M.C. and Hayes, A.W. (2016) Advancing risk assessment through the application of 
systems toxicology. Toxicol. Res., 32, 5-8.

3. Casey, W.M. (2016) Advances in the development and validation of test methods in the united states. Toxicol. Res., 32, 914.

4. Leist, M., Hasiwa, N., Rovida, C., Daneshian, M., Basketter, D., Kimber, I., Clewell, H., Gocht, T., Goldberg, A., Busquet, F., Rossi, A.M., Schwarz, M., Stephens, M., Taalman, R., Knudsen, T.B., McKim, J., Harris, G., Pamies, D. and Hartung, T. (2014) Consensus report on the future of animal-free systemic toxicity testing. Altex, 31, 341-356.

5. Yoon, M. and Clewell, H.J. III (2016) Addressing early life sensitivity using physiologically based pharmacokinetic modeling and in vitro to in vivo extrapolation. Toxicol. Res., 32, 15-20.

6. Harada, T., Takeda, M., Kojima, S. and Tomiyama, N. (2016) A review of toxicity and carcinogenicity of dichlorodiphenyltrichloroethane (DDT) with special reference to hepatocarcinogenesis. Toxicol. Res., 32, 21-33.

7. Chun, S.K., Go, K., Yang, M.-J., Zendejas, I. Behrns, K.E. and Kim, J.-S. (2016) Autophagy in ischemic livers: A critical role of sirtuin $1 /$ mitofusin 2 axis in autophagy induction.
Toxicol. Res., 32, 35-46.

8. Cheon, J.H., Kim, S.Y., Son, J.Y., Kang, Y.R., An, J.H., Kwon, J.H., Song, H.S., Moon, A., Lee, B.M. and Kim, H.S. (2016) Pyruvate kinase M2: a new biomarker for the early detection of acute kidney injury. Toxicol. Res., 32, 47-56.

9. Lim, H., Lim, J.-a., Choi, J., Kwon, H.-j., Ha, M., Kim, H. and Park, J.-d. (2016) Association between multiple metal exposure and renal impairment in Korean population. Toxicol. Res., 32, 57-64.

10. Chunhabundit, R. (2016) Cadmium exposure and potential health risk from foods in contaminated area, Thailand. Toxicol. Res., 32, 65-72.

11. Lee, J.-Y., Tokumoto, M., Hattori, Y., Fujiwara, Y., Shimada, A. and Satoh, M. (2016) Different regulation of p53 expression by cadmium exposure in kidney, liver, intestine, vasculature, and brain astrocytes. Toxicol. Res., 32, 73-80.

12. Bintvihok, A., Treebonmuang, S., Srisakwattana, K., Nuanchun, W., Patthanachai, K. and Usawang, S. (2016) A rapid and sensitive detection of aflatoxin-producing fungus using an optimized polymerase chain reaction (PCR). Toxicol. Res., 32, 81-87. 